Review Article www.pjkd.com.pk

\title{
Uremic Cardiomyopathy
}

\author{
Amna Umer', M. Azhar Waheed Khan', Maryam Javed², Azhar Ali Khan ${ }^{3}$ \\ ${ }^{1}$ Avicenna Medical College, Lahore, Pakistan. \\ ${ }^{2}$ Sheikh Zayed Hospital, Lahore, Pakistan. \\ ${ }^{3}$ Azra Naheed medical College, Lahore, Pakistan.
}

\begin{abstract}
Chronic Kidney Disease (CKD) affects approximately 10\% of the general population. CKD itself is a risk factor for cardiovascular mortality and morbidity. Large epidemiological studies have clearly established a clear relationship between severity of CKD and cardiovascular event rates. Sudden cardiac death accounts for approximately $40 \%$ mortality in dialysis patients and it is usually secondary to an underlying cardiomyopathy, with left ventricular hypertrophy mostly evident on echocardiography. Several risk factors play an important role in pathogenesis of uremic cardiomyopathy including vitamin D deficiency, secondary hyperparathyroidism, phosphate retention, increased FGF23 and decreased Klotho levels. The mortality due to cardiovascular events in early CKD is relatively higher in comparison to progression to ESKD, so early diagnosis and treatment with dialysis should be focused.
\end{abstract}

Keywords: End stage renal disease, sudden cardiac death, hemodialysis, cardiomyopathy, uremia.

\section{Corresponding Author}

Dr. Azhar Ali Khan

Assistant Professor

Consultant Nephrologist

Azra Naheed Medical College

azhar_aq@hotmail.com

Received: February 2,2021. Accepted March 06, 2021.

PJKD 2021;5(1):35-38

\section{Introduction}

The term chronic kidney disease encompasses all degrees of decreased renal function from earliest stages to end stage kidney disease (ESKD) and it has a rapidly increasing prevalence. The traditional risk factors includes age hypertension, dyslipidemia, obesity and diabetes whereas nontraditional risk factors include uremia, anemia and hypervolemia. ${ }^{1}$ Echocardiography is the most common method for studying structural abnormalities of uremic cardiomyopathy which shows that left ventricular hypertrophy is the prominent feature. Park et al study shows a graded relationship between the severity of CKD and LVH along with its prevalence. ${ }^{2}$ A study using MRI with gadolinium contrast showed evidence of myocardial fibrosis in uremic patients that was different in distribution to the sub endocardial fibrosis observed in ischemic heart disease. ${ }^{3}$ Moreover, histopathological examination of postmortem cardiac tissues samples of hemodialysis patients showed increased cardiomyocyte diameter, reduced capillary length density and increased interstitial volume. 


\section{Uremic Cardiomyopathy}

\section{Risk Factors}

Important risk factors that contribute to left ventricular hypertrophy include hypertension, volume overload and anemia. Several risk factors specific to the pathogenesis of uremic cardiomyopathy are discussed here.

Vitamin D: Vitamin D deficiency modifies cardiac structure and function thus leading to decreased cardiovascular related morbidity and mortality as reported by Thandhani et al. 48 weeks study using cardiovascular magnetic resonance showed that Vitamin D does not affect left ventricular mass and it does not improve diastolic dysfunction in CKD patients. ${ }^{4}$

Tryptophan \& Tyrosine Metabolites: Indoxyl sulfate (IS) and p-cresyl sulfate (p-CS) comes from bacterial protein metabolism of tryptophan and tyrosine in large intestine. ${ }^{5}$ These compounds circulate non-covalently bound to albumin so they are not easily filtered. In patients who have normal GFR, the circulating levels of IS are $\sim 0.5 \mathrm{mg} / \mathrm{L}$ and in uremia they are up to $\sim 20 \mathrm{mg} / \mathrm{L}$. Lekawanvijit et.al study showed that IS/p-CS was involved in causing complications that were related to uremia, including oxidative stress, fibrosis, inflammation and cardiac dysfunction. ${ }^{6}$ Several studies have shown a relationship between hyperphosphatemia in CKD with cardiovascular mortality.

FGF 23 and Hyperphosphatemia: In patients undergoing regular hemodialysis had a significant association of hyperphosphatemia and hyperparathyroidism with cardiovascular related hospitalization. It is thought to be related to vascular calcification that contributes to hypertrophy of the heart.

FGF23 is a hormone that is derived from bone, where it is secreted mainly by osteocytes. FGF23 blocks sodium dependent phosphorus co-transporter in the proximal convoluted tubule causing decreased phosphate reabsorption and increased phosphate excretion. ${ }^{8}$ In addition, FGF23 inhibits $1 \alpha$-hydroxylase and increases 24-hydroxylase activity therefore, suppresses 1, 25dihydroxyvitamin D synthesis leading to decreased phosphate absorption from the gut. By decreasing PTH secretion from parathyroid gland, FGF23 decreases the resorption of bone and hence phosphate release into the circulation. For maintaining phosphorus hemostasis in CKD, FGF23 production from bone is increased in order to decrease phosphate absorption from intestine and phosphate release from bones and ultimately to cause phosphaturia.

Therefore, in patients with CKD, increased level of FGF23 is an adaptive response that fights against phosphate retention. Recent studies show that this compensatory increase in FGF23 causes increase in mortality by directly inducing LVH. This hypothesis is supported by positive correlation between FGF23 levels and LVH in dialysis patient.' The study of Garbner et al showed that the action of FGF23 on heart was via 4FGF receptor and $\mathrm{PLC} \gamma /$ Calcineurin /NFAT pathway that induce hypertrophy. The study also shows that blocking the FGFR4 receptor with monoclonal antibodies protect against FGF23-induced LVH, without loss of physiological effects of FGF23. ${ }^{10}$ In general, FGF23 plays a very important role in CKD related cardiomyopathy and myocardial FGFR4 blockade seems to be an auspicious therapeutic target for its prevention. 


\section{Uremic Cardiomyopathy}

Klotho: Klotho serves as a critical co-factor for FGF23 in kidneys and parathyroid gland. CKD is a state of klotho deficiency that leads to FGF23 resistance. Decreased klotho expression in the kidney inhibits phosphaturia effects of FGF23 leading to increased phosphate levels in the blood. Klotho prevents the hypertrophy of heart by down regulation of calcium permeable channels in heart (11). Calcium entry through TRPCG channels activates Calcineurin and nuclear factor of activated T cells (NFAT) signaling cascade which plays a pivotal role in pathogenesis of pathological cardiac hypertrophy. ${ }^{12}$ Therefore, decreased level of klotho in circulation contributes to uremic cardiomyopathy.

$\mathrm{LVH}$ is an independent predictor of survival in $\mathrm{CKD}$, moreover, regression of $\mathrm{LVH}$ leads to decreased mortality associated with cardiovascular complication. ${ }^{13,14}$ Conventional hemodialysis is the most commonly used treatment option for uremic cardiomyopathy and it is more effective if started early in the disease process before any irreversible damage to myocardium occurs. For the dialysis to be effective, the duration and frequency of hemodialysis is very important in order to optimize ultrafiltration rate. ${ }^{15,16}$ Some studies show that peritoneal dialysis is more effective in uremic cardiomyopathy because it allows gentle ultrafiltration but a cohort study from 1995 to 1997 shows that rate of mortality is higher for the patient with uremic cardiomyopathy undergoing peritoneal dialysis. ${ }^{17}$ The best treatment so far is short hemodialysis in which a patient receive dialysis for 5 6 days a week and for 1.5 to $2 \mathrm{hrs}$., duration in each session, ultimately leading to decrease in $\mathrm{LVH}^{18}$ Dzemidzic et.al showed that after successful renal transplant LVH can decrease from $67-37 \%$ within first year post transplant. ${ }^{19}$ It confirms that elimination of uremia risk factors decrease LVH. Renal transplant provides significant survival advantage over hemodialysis, counseling regarding renal transplant should be done in patients with ESKD as soon as the diagnosis of systolic heart dysfunction is made. ${ }^{20}$

\section{Conclusion}

Uremic cardiomyopathy, characterized by left ventricular Hypertrophy, is the major cause of cardiovascular mortality in CKD patients. The role of hyperphosphatemia, high levels of FGF23 and deficiency of $\alpha$ klotho are mainstay to the pathophysiology of cardiomyopathy in CKD patients. The early initiation of hemodialysis may slow the progression of uremic cardiomyopathy. Conventional hemodialysis is superior to non-conventional. It has been observed that renal transplantation reverses uremic cardiomyopathy and has a survival benefit over hemodialysis.

\section{References}

1. Coresh J, et al. Prevalence of chronic kidney disease in the United States. JAMA. 2007;298:2038-47.

2. Park M, Hsu CY,Li y, et al. Associations beween kidney function and subclinical cardiac abnormalities in CKD. J Am Soc Nephrol 2012;23(10):1725-34

3. Mark PB, Johnston N, Groenning BA, et al. Redefinition of uremic cardiomyopathy by contrast-enhanced cardiac magnetic resonance imaging. Kidney Int 2006;69(10):1839-45

4. Thadhani R, et al. Vitamin $D$ therapy and cardiac structure and function in patients with chronic kidney disease: the PRIMO randomized controlled trial. JAMA. 2012; 307: 674-84.

5. Meijers BK, Evenepoel P.The gut-kidney axis: indoxyl sulfate, p-cresyl sulfate and CKD progression. Nephrol Dial Transplant. 2011; 26: 759-61.

6. Lekawanvijit S, Kompa AR, Wang BH, Kelly DJ, Krum H. Cardiorenal syndrome: the emerging role of protein-bound uremic toxins. Circ Res. 2012; 111: 1470-83.

7. Block GA, Hulbert-Shearon TE, Levin NW, Pork FK. Association of serum phosphorus and calcium X phosphate product with mortality risk in chronic hemodialysis patients: a national study. Am J Kidney Dis 1998; 31: 607-17 


\section{Uremic Cardiomyopathy}

8. Saito T, Fukumoto S. Fibroblast Growth Factor 23 (FGF23) and Disorders of Phosphate Metabolism. Int J Pediatr Endocrinol. 2009:496514.

9. Faul C, et al. FGF23 induces left ventricular hypertrophy. J Clin Invest. 2011; 121:4393-4408

10. Grabner A, Amaral AP, Schramm K, Singh S, Sloan A, Yanucil C, et al. Activation of cardiac fibroblast growth factor receptor 4 causes left ventricular hypertrophy. Cell Metab. 2015;22:1020 - 32.

11. Xie J, Cha S-K, An S-W, Kuro-o M, Birnbaumer L, Huang C-L. Cardioprotection by Klotho through downregulation of TRPC6 channels in the mouse heart. Nat. Commun. 2012; 3: 1238.

12. Kuwahara K, et al. TRPC6 fulfills a calcineurin signalling circuit during pathologic cardiac remodeling. J Clin Invest. 2006;116:3114 - 3126.

13. Silberberg JS, Barre PE, Prichard SS, Sniderman AD. Impact of left ventricular hypertrophy on survival in end-stage renal disease. Kidney Int. 1989;36:286 - 290.

14. London GM, Pannier B, Guerin AP, et al. Alterations of left ventricular hypertrophy in and survival of patients receiving hemodialysis: follow-up of an interventional study. J Am Soc Nephrol. 2001;12:2759-27673

15. Burton JO, Jefferies HJ, Selby NM, McIntyre CW. Hemodialysisinduced cardiac injury: determinants and associated outcomes. Clin J Am Soc Nephrol. 2009;4:914 - 920.

16. Stack AG, Molony DA, Rahman NS, Dosekun A, Murthy B. Impact of dialysis modality on survival of new ESRD patients with congestive heart failure in the United States. Kidney Int. 2003;64:1071-79

17. Fagugli R, Reboldi G, et al. Short daily hemodialysis: blood pressure control and left ventricular mass reduction in hypertensive hemodialysis patients. Am J Kidney Dis. 2001;38:371 - 376

18. Ayus JC, Achinger SG, Thadhani R. Effects of short daily versus conventional hemodialysis on left ventricular hypertrophy and inflammatory markers: a prospective, controlled study. J Am Soc Nephrol. 2005;16:2778-88

19. Dzemidzic J, Rasic S, Saracevic A, et al. Predictors of left ventricular remodeling in kidney transplant recipients in the first posttransplant year. Bosn J Basic Med Sci. 2010;10(suppl 1):S51 - S55

20. Wali RK, Wang GS, Gottlieb SS, et al. Effect of kidney transplantation on left ventricular systolic dysfunction and congestive heart failure in patients with end-stage renal disease. J Am Coll Cardiol. 2005;45:1051 - 1060. 